\title{
Ajuste psicosocial en adolescentes víctimas frecuentes y víctimas ocasionales de violencia de pareja
}

\author{
Psychosocial Adjustment in occasional and frequent Victims of teen Dating Violence
}

\author{
Laura Carrascosa \\ María-Jesús Cava \\ Sofía Buelga \\ Universidad de Valencia, España
}

Rec (08 octubre 2015) Acept (25 junio 2016)

\begin{abstract}
Resumen
El objetivo de esta investigación es analizar el ajuste psicosocial en los adolescentes víctimas de violencia de pareja, considerando posibles diferencias en función de la frecuencia de la victimización y del sexo de los adolescentes. Los indicadores del ajuste psicológico y social analizados son el ánimo depresivo, los sentimientos de soledad, el autoconcepto, la satisfacción con la vida y la calidad de la comunicación con el padre y la madre. Participaron 672 adolescentes españoles (325 chicas, 347 chicos), entre 12 y 19 años $(M=14.45 ; D T=1.62)$. Los resultados indicaron que los adolescentes, chicos y chicas, en situación frecuente de victimización presentan menor autoconcepto familiar, más problemas de comunicación con la madre, mayor ánimo depresivo y sentimientos de soledad y menor satisfacción con la vida que los adolescentes cuya victimización es ocasional. Estos resultados muestran la necesidad de diferenciar entre ambos grupos de adolescentes víctimas de la violencia de pareja.

Palabras clave: violencia en parejas adolescentes, victimización, ajuste psicosocial
\end{abstract}

\begin{abstract}
The objective of this research is to analyze the psychosocial adjustment on adolescent victims of dating violence, considering possible differences in terms of the frequency of victimization and sex of adolescents. The analyzed indicators of psychological and social adjustment are depressed mood, feelings of loneliness, self-concept, satisfaction with life and quality of communication with father and mother. 672 Spanish adolescents ( 325 girls, 347 boys), aged 12 to 19 years $(M=14.45, S D=1.62)$ participated. The results indicated that adolescents, boys and girls, frequently victimized have less family self-concept, more communication problems with the mother, more depressive mood and feelings of loneliness and less satisfaction with life, than adolescents whose victimization is occasional. These results show the need to differentiate between both groups of adolescent victims of dating violence.

Keywords: teen dating violence, victimization, psychosocial adjustment
\end{abstract}

\footnotetext{
Esta investigación se ha realizado dentro del proyecto de investigación PSI2012-33464 "La violencia escolar, de pareja y filio-parental en la adolescencia desde la perspectiva ecológica", subvencionado por el Ministerio de Economía y Competitividad de España.

Correspondencia: Laura Carrascosa, Universitat de València. Facultat de Psicologia Avda. Blasco Ibáñez, 21. 46010. Valencia. Tfno:963983492. Email: carrascosa.iranzo@gmail.com; María-Jesús Cava. Persona de contacto Universitat de València. Facultat de Psicologia Avda. Blasco Ibáñez, 21. 46010. Valencia. Tfno:963983492. Email: maria.j.cava@uv.es; Sofía Buelga, Universitat de València. Facultat de Psicologia Avda. Blasco Ibáñez, 21. 46010. Valencia. Tfno:963864575. Email: sofia.buelga@uv.es
} 


\section{Introducción}

La violencia en parejas adolescentes constituye una importante problemática, aunque ha sido menos investigada que la violencia en parejas adultas (Cortés-Ayala, Flores, Bringas, Rodríguez-Franco, López-Cepero y Rodríguez, 2015; Leen et al., 2013; Rey-Anacona, Mateus-Cubides y Bayona-Arévalo, 2010; Viejo, 2014). Esta violencia presenta ciertas diferencias respecto a la violencia en parejas adultas, constatándose una mayor frecuencia de la violencia verbal y emocional, así como de las agresiones mutuas (FernándezFuertes y Fuertes, 2010; Foshee y Reyes, 2011; Muñoz-Rivas, Graña, O’Leary y González, 2007). Para los adolescentes las primeras relaciones de pareja conllevan nuevos retos y nuevas formas de relacionarse, que suelen contribuir positivamente a su desarrollo psicosocial (Furman, Low y Ho, 2009). Sin embargo, su falta de experiencia previa se ha relacionado con su implicación, en algunos casos, en formas torpes o bruscas de cortejo que podrían incluir algunas conductas violentas de acercamiento y que podrían explicar, al menos en parte, la elevada prevalencia de la violencia en parejas adolescentes (Ortega y Sánchez, 2011; Viejo, 2014; Viejo, Monks, Sánchez y Ortega-Ruiz, 2015).

Ciertamente, una de las características de la violencia de pareja en adolescentes es su elevada prevalencia, señalada en estudios realizados en diversos países como Estados Unidos (Cornelius y Resseguie, 2007; O'Leary, Slep, Avery-Leaf y Cascardi, 2008), México (Cortés-Ayala et al., 2015), Colombia (Rey-Anacona, 2013) o España (FernándezFuertes y Fuertes, 2010; Muñoz-Rivas et al., 2007). En el estudio realizado en España por Muñoz-Rivas et al. (2007) un elevado porcentaje de adolescentes (un $95.3 \%$ de las chicas y un $92.8 \%$ de los chicos) indicó haber agredido verbalmente alguna vez a su pareja; siendo menor, aunque también considerable, el porcentaje de adolescentes (un 2\% de los chicos y un $4.6 \%$ de las chicas) que indicaron haber agredido físicamente alguna vez a su pareja. En un estudio posterior realizado por Fernández-Fuertes y Fuertes (2010) se obtuvieron datos de prevalencia similares, con más de un 94\% de adolescentes (chicos y chicas) que señalaron haber agredido y haber sido víctimas de alguna agresión verbal en su relación de pareja.

En los dos estudios citados realizados en España (Fernández-Fuertes y Fuertes, 2010; Muñoz-Rivas et al., 2007), al igual que en la mayoría de investigaciones sobre prevalencia de la violencia en parejas adolescentes, únicamente se ha tenido en cuenta la presencia o ausencia de determinadas conductas violentas en la pareja, aunque la frecuencia de estas conductas podría ser también un aspecto relevante. Así, en algunas parejas las conductas violentas son ocasionales y, generalmente, de escasa gravedad. Estas conductas podrían estar, en cierto modo, relacionadas con algunas formas torpes y bruscas de acercamiento a la pareja y de cortejo (Ortega y Sánchez, 2011; Viejo, 2014). En otras parejas las conductas violentas son frecuentes y podrían ser indicativas de mayores dificultades de adaptación en estos adolescentes e incluso estar vinculadas a experiencias de violencia y de victimización en otros ámbitos, como el familiar o el de las relaciones entre iguales (Cuevas, Sabina y Bell, 2014).

Diversos estudios han relacionado las situaciones de victimización de pareja en adolescentes con un peor ajuste psicosocial (Exner-Cortens, Eckenrode y Rothman, 2013; Leen et al., 2013). Los adolescentes, chicos y chicas, víctimas de violencia de pareja muestran menor satisfacción con la vida, bajo rendimiento académico, niveles elevados de estrés, ansiedad, sintomatología depresiva, quejas somáticas, abuso de sustancias, trastornos alimenticios y conductas sexuales de riesgo (Jouriles, Garrido, Rosenfield y McDonald, 2009; Romito, Beltramini y Escribà-Agüir, 2013; Shorey, Rhatigan, Fite y Stuart, 2011; Saldivia y Vizcarra, 2012; Shorey et al., 2015). Estas dificultades en su ajuste psicosocial podrían ser mayores o menores dependiendo de la frecuencia con la que la violencia está presente en su relación de pareja. A este respecto, se ha constatado que en las relaciones de pareja en adolescentes en las que la violencia es frecuente coexisten diferentes tipos de violencia y las agresiones son de mayor gravedad (Cuevas et al., 2014; Foshee y Reyes, 2011; Leen et al., 2013; Sears, Byers y Price, 2007).

Aunque ciertamente la presencia o ausencia de violencia en la pareja es un factor clave y la violencia es siempre un elemento a eliminar en todas las relaciones, una mejor comprensión de la violencia en parejas adolescentes parece requerir el análisis de la frecuencia con que se producen las conductas violentas, tal y como ha sido sugerido en algunos estudios previos (Rey-Anacona, 2013). El ajuste psicológico y social de los adolescentes que son víctimas frecuentes de violencia de pareja podría ser diferente de aquellos adolescentes cuya victimización es ocasional. En este sentido, algunos de los indicadores que convendría comparar en ambos grupos son la soledad, el ánimo depresivo y la satisfacción con la vida. Aunque en estudios previos se ha relacionado la victimización en parejas adolescentes con mayor ánimo depresivo y menor satisfacción con la vida (Romito et al., 2013; Shorey et al., 2015), podrían existir diferencias en función de la frecuencia de la victimización. En cuanto a 
la soledad, su relación con la victimización de pareja no ha sido analizada en adolescentes. No obstante, es previsible que ambas variables estén relacionadas, ya que la pareja y los iguales son fundamentales para los adolescentes, y las dificultades con los iguales se han vinculado a mayores sentimientos de soledad (Povedano, Cava, Monreal, Varela y Musitu, 2015).

Otro importante indicador de ajuste psicosocial a considerar es el autoconcepto, puesto que durante la adolescencia se producen cambios importantes en la imagen que la persona tiene de sí misma a nivel físico, social y familiar (Coleman y Hendry, 2003). Actualmente, se asume una perspectiva multidimensional del autoconcepto y se considera integrado por diferentes dimensiones relacionadas con distintos ámbitos, como el familiar, académico, social, emocional o físico (García, Musitu, Riquelme y Riquelme, 2011). En los adolescentes se han encontrado correlaciones positivas entre autoconcepto académico y rendimiento escolar (GonzálezPienda et al., 2003) y un autoconcepto social positivo se ha relacionado con menor victimización de los iguales (Estévez, Martínez y Musitu, 2006) y mayor sensibilidad intercultural (Micó-Cebrían y Cava, 2014). Un adecuado autoconcepto familiar se considera un factor de protección ante conductas de riesgo en adolescentes (Cava, Murgui y Musitu, 2008), por tanto, sería interesante analizar las relaciones entre sus distintas dimensiones y las situaciones de victimización en parejas adolescentes.

Por último, es también necesario incluir variables indicativas del funcionamiento familiar, puesto que la familia es esencial en el desarrollo del adolescente y se ha constatado que las relaciones familiares basadas en la cohesión, la adecuada comunicación y el apoyo mutuo favorecen el ajuste y bienestar psicosocial de los hijos (Cava, Buelga y Musitu, 2014). En las familias con límites claros, afectivas y con una comunicación positiva y abierta, la implicación de los hijos en situaciones de violencia de pareja es menor (Vézina y Hérbert, 2007). Asimismo, la supervisión de los padres y su cercanía afectiva se han señalado como factores de protección ante la victimización de pareja (Foshee et al., 2015; Kast, Eisenberg y Sieving, 2015).

Teniendo en cuenta la importancia de estos indicadores, el objetivo de esta investigación es analizar las diferencias en ánimo depresivo, sentimientos de soledad, satisfacción con la vida, autoconcepto (social, físico, académico, familiar y emocional) y comunicación con la madre y el padre en adolescentes que sufren situaciones de violencia de pareja, teniendo en cuenta posibles diferencias en función de la frecuencia de la victimización y del sexo del adolescente.
Como hipótesis iniciales se plantea, en primer lugar, que los adolescentes victimizados de forma frecuente tendrán peor ajuste psicosocial que los adolescentes que no sufren victimización y que los victimizados de forma ocasional. En segundo lugar, se plantea que la mayor frecuencia de victimización se relacionará con peor ajuste tanto en chicos como en chicas.

\section{Método}

\section{Participantes}

La muestra inicial estuvo compuesta por 1.078 adolescentes (545 chicas y 533 chicos), entre 12 y 19 años de edad $(M=14.45, D T=1.62)$, estudiantes de Educación Secundaria y Bachillerato en cuatro centros educativos de la región de Valencia (España), tres de ellos públicos y uno privado-concertado. Un $20.50 \%$ estudiaba primer curso de Educación Secundaria, 24.30\% estudiaba segundo curso, $22.20 \%$ tercer curso, $20.90 \%$ cuarto curso y un $12.10 \%$ cursaba estudios de Bachillerato. Todos los adolescentes participantes cumplimentaron los instrumentos que evalúan las variables de ajuste psicosocial, como parte de un estudio más amplio sobre la adolescencia. No obstante, solo los chicos y chicas adolescentes que tenían pareja en ese momento o que la habían tenido en los últimos 12 meses (325 chicos y 347 chicas) cumplimentaron el instrumento que evalúa la victimización de pareja.

La muestra final de este estudio consta, por tanto, de 672 adolescentes, 325 chicos (48.4\%) y 347 chicas (51.6\%). Durante la cumplimentación de la escala de victimización de pareja se les indicó a los adolescentes que pensaran en su última relación de pareja, caso de haber tenido varias durante los últimos 12 meses. Su rango de edad se situó entre 12 y 19 años $(M=14.45 ; D T=1.62)$, siendo similar la edad media de chicos $(M=14.36 ; D T=1.48)$ y chicas $(M=14.70 ; D T=1.65)$. Los porcentajes de adolescentes con 13 años (20.6\%), 14 años (20.9\%) y 15 años (22.0\%) son los más elevados, siendo sensiblemente menor el porcentaje de adolescentes con 12 años (9.2\%) y con 18 y 19 años $(3.8 \%)$.

\section{Instrumentos}

Escala de Conflicto en Parejas Adolescentes CADRI (Conflict in Adolescent Dating Relationships Inventory de Wolfe, Scott, Reitzel-Jaffe, Wekerle, Grasley y Pittman, 
2001; adaptación española de Fernández-Fuertes, Fuertes y Pulido, 2006). La escala original consta de 70 ítems que evalúan tanto comportamientos violentos hacia la pareja como experiencias de victimización. En este estudio se utilizaron únicamente tres subescalas que evalúan diferentes tipos de victimización en la pareja: Victimización física, compuesta por 4 ítems (por ejemplo, "mi pareja me empujó o me zarandeó") que evalúan comportamientos violentos de tipo físico, tales como golpes o empujones; Victimización verbal-emocional, que consta de 10 ítems (por ejemplo, "mi pareja me insultó con frases de desprecio") relativos a amenazas, insultos o chantajes ejercidos por la pareja; y Victimización relacional, compuesta por 3 ítems (por ejemplo, "mi pareja trató de apartarme de mi grupo de amigos") que evalúan el daño recibido a través de conductas como la difusión de rumores o el control de las relaciones. En estos ítems los adolescentes deben identificar con qué frecuencia se han producido en la relación con su pareja las situaciones descritas: nunca -esto no ha pasado en nuestra relación-, rara vez -1 o 2 ocasiones-, a veces -entre 3 y 5 veces- o con frecuencia -6 o más ocasiones-. $\mathrm{Su}$ fiabilidad (alpha de Cronbach) en este estudio fue .82 Victimización física, .85 Victimización verbal-emocional y .64 Victimización relacional. La fiabilidad de la escala total de Victimización fue 91 .

Escala de Autoconcepto-AF5-(García y Musitu, 1999; García et al., 2011). Esta escala consta de 30 ítems y mide cinco dimensiones del autoconcepto: Autoconcepto académico, relativo al concepto que tiene el adolescente de sí mismo en el ámbito académico y escolar (por ejemplo: "Hago bien los trabajos escolares"); Autoconcepto social, relativo al concepto de sí mismo a nivel social (por ejemplo: "Consigo fácilmente amigos/as"); Autoconcepto emocional, concepto propio sobre la capacidad de mantener estabilidad emocional ante situaciones nuevas o difíciles (por ejemplo: "Tengo miedo de algunas cosas"); Autoconcepto familiar, referido al concepto de sí mismo en el ámbito familiar (por ejemplo: "Me siento querido/a por mis padres"); y Autoconcepto físico, relativo al ámbito físico (por ejemplo: "Me buscan para hacer actividades deportivas"). A estos ítems se responde mediante 5 opciones de respuesta, desde 1 -nunca-hasta 5-siempre-. Su consistencia interna (alpha de Cronbach) en este estudio fue .89 Autoconcepto académico; .71 Autoconcepto social; .68 Autoconcepto emocional; .80 Autoconcepto familiar y .77 Autoconcepto físico.

Escala de Comunicación Padres-Adolescentes (Barnes y Olson, 1985; adaptación de Jiménez, Estévez, Musitu y Murgui, 2007). Consta de dos escalas: una relativa a comunicación con la madre (desde la perspectiva del hijo/a) y otra referida a la comunicación con el padre (también desde la perspectiva del hijo/a). Ambas escalas tienen 20 ítems, agrupados en 2 dimensiones: Comunicación abierta y Problemas en la comunicación. La Comunicación abierta consta de 11 ítems que hacen referencia a una comunicación positiva entre padres e hijos, basada en la libertad, comprensión y libre intercambio de información (por ejemplo, "Me presta atención cuando le hablo"). La dimensión de Problemas en la comunicación consta de 9 ítems relativos a una comunicación negativa y basada en la crítica (por ejemplo, "Intenta ofenderme cuando se enfada conmigo"). El rango de respuesta oscila entre 1 -nunca- y 5 -siempre-, tanto en comunicación con el padre como con la madre. La fiabilidad (alpha de Cronbach) en esta investigación para las subescalas de Comunicación abierta con el padre y la madre fue .91 para ambas; y para Problemas en la comunicación con el padre y la madre .70, para ambas.

Escala de Satisfacción con la Vida (Diener, Emmons, Larsen y Griffin, 1985; adaptación de Atienza, Pons, Balaguer y García-Merita, 2000). Consta de 5 ítems que miden mediante una escala de cinco puntos, desde $1-$ - mиy en desacuerdo- hasta 5-muy de acuerdo-, el grado de satisfacción de las personas con su vida (por ejemplo: "Si pudiera vivir mi vida otra vez, me gustaría que fuera como ha sido hasta ahora"). Su fiabilidad (alpha de Cronbach) en esta investigación fue .76.

Escala de Sintomatología Depresiva-CES-D-(Radloff, 1977; adaptación de Herrero y Meneses, 2006). Esta escala consta de 7 ítems (por ejemplo, "me costaba concentrarme en lo que estaba haciendo") que evalúan con un rango de respuesta desde 1 -nunca- hasta 4-siempre-varias dimensiones relativas a sintomatología depresiva (ánimo deprimido, desamparo y desesperación, pérdida de apetito, problemas de sueño y problemas de concentración). No evalúa la depresión en sí misma, sino la sintomatología asociada y proporciona un índice general de ánimo depresivo. Su fiabilidad (alpha de Cronbach) en esta investigación fue 80 .

Escala de Soledad-UCLA-(Russell, Peplau y Cutrona, 1980, adaptación de Expósito y Moya, 1999. Consta de 20 ítems con cuatro opciones de respuesta, desde $1-n u n c a-a$ 4 -siempre-. Aunque originalmente se consideró integrada por un único factor, que aportaba un índice general de soledad percibida, en estudios posteriores (Borges, Prieto, Ricchetti, Hernández-Jorge y Rodríguez-Naveiras, 2008) se ha obtenido una estructura bifactorial: Soledad emocional, que consta de 11 ítems (por ejemplo, "con qué frecuencia sientes que te falta compañía") y Evaluación subjetiva de la red social, conformada por 9 ítems (por ejemplo, "con qué frecuencia piensas que hay gente a quien puedes pedir 
ayuda"). Esta estructura bifactorial fue confirmada en esta investigación: el primer factor, Soledad emocional, explicó un $30.21 \%$ de varianza y el segundo, Evaluación de la red social, un 6.65\%. La fiabilidad de ambos factores (alpha de Cronbach) fue muy buena, con valores de .84 Soledad emocional y .86 Evaluación de la red social.

\section{Procedimiento}

La selección de la muestra se realizó mediante muestreo por conglomerados. Las unidades de muestreo fueron los centros educativos de Enseñanza Secundaria y Bachillerato de la región de Valencia (España). Una vez seleccionados los centros, se contactó telefónicamente para solicitarles una entrevista y explicarles los objetivos y procedimiento de la investigación. Se realizó una reunión con el equipo directivo y con el profesorado para explicar el proyecto de investigación y solicitar su colaboración. Los cuatro centros seleccionados aceptaron participar en el estudio. Posteriormente, se envió a los padres una carta informativa en relación con este proyecto y se les pidió su consentimiento para la participación de sus hijos. Una vez obtenidos los permisos, varios investigadores previamente entrenados realizaron el pase de los instrumentos en las aulas. Los adolescentes fueron informados de que su participación era voluntaria y anónima. El estudio cumplió los valores éticos requeridos en la investigación con seres humanos, respetando los principios fundamentales de la Declaración Helsinki, en sus actualizaciones y normativas vigentes (consentimiento informado y derecho a información, protección de datos personales y garantías de confidencialidad, no discriminación y posibilidad de abandonar el estudio en cualquiera de sus fases).

\section{Análisis de datos}

Para analizar el ajuste psicosocial de los adolescentes víctimas de violencia de pareja, diferenciando entre victimización ocasional y frecuente, se establecieron tres grupos en función de sus puntuaciones en la escala de victimización de pareja. En primer lugar, se calcularon las puntuaciones totales de todos los adolescentes sumando sus puntuaciones en las tres subescalas de victimización. El rango de puntuaciones posibles oscila entre 17 (contestando "nunca" -valor asignado 1- a los 17 ítems) y 68 (contestando "con frecuencia" -valor asignado 4- a los 17 ítems). Las puntuaciones en esta investigación oscilaron entre 17 y 60 (puntuación media $=21.98$, desviación típica $=6.48$ ). Una vez obtenidas sus puntuaciones totales, se estableció un primer grupo denominado "sin victimización" integrado por los adolescentes cuyas puntuaciones eran iguales a 17, es decir, aquellos adolescentes que contestaron "nunca" en todos los ítems. Este grupo quedó conformado por 185 adolescentes (107 chicos, 78 chicas).

En segundo lugar, se diferenció entre los adolescentes cuyas puntuaciones totales superaban la media en 1 desviación típica de aquellos cuyas puntuaciones no superaban este punto de corte, un criterio que también han seguido otros estudios sobre violencia en adolescentes para establecer diferencias en frecuencia e intensidad (Buelga, Iranzo, Cava y Torralba, 2015). Los adolescentes con puntuaciones superiores a la media en 1 desviación típica (puntuaciones $>28.46$ ) fueron asignados al grupo de "victimización frecuente" y los adolescentes con puntuaciones superiores a 17 , pero inferiores a este punto de corte, al grupo de "victimización ocasional". El grupo de "victimización frecuente" estuvo compuesto por 81 adolescentes (48 chicos, 33 chicas) y el de "victimización ocasional" por 394 (163 chicos, 231 chicas).

Posteriormente, se realizó un MANOVA 3 x 2, para analizar posibles diferencias en Autoconcepto, Ánimo depresivo, Satisfacción con la vida, Soledad, Comunicación con la madre y Comunicación con el padre en función de los tres grupos establecidos ("sin victimización", "victimización ocasional" y "victimización frecuente") y del sexo (chico-chica) de los adolescentes, así como de la interacción entre grupo y sexo. Todos los análisis se realizaron con el paquete estadístico SPSS-20.

\section{Resultados}

Los resultados del MANOVA realizado indicaron diferencias estadísticamente significativas entre los tres grupos con diferente nivel de victimización (Lambda de Wilks= $\left..832 ; \mathrm{F}_{26,1106}<.001\right)$ y entre chicos y chicas (Lambda de Wilks $\left.=.744 ; \mathrm{F}_{13,553}<.001\right)$, pero no en la interacción entre ambas variables (Lambda de Wilks $=.956 ; \mathrm{F}_{26,1106}>.05$ ).

Para llevar a cabo un análisis más detallado se realizaron pruebas de efectos inter-sujetos (Tabla 1). Los resultados mostraron que los adolescentes con diferente nivel de victimización diferían en Autoconcepto académico $\left(\mathrm{F}_{2,565}\right.$ $=5.191, p<.01)$, Autoconcepto social $\left(\mathrm{F}_{2,565}=5.803, p\right.$ $<.01)$, Autoconcepto emocional $\left(\mathrm{F}_{2,565}=4.237, p<.05\right)$, Autoconcepto familiar $\left(\mathrm{F}_{2,565}=9.860, p<.001\right)$, Autoconcepto físico $\left(\mathrm{F}_{2,565}=3.291, p<.05\right)$, Comunicación abierta con la madre $\left(\mathrm{F}_{2,565}=3.206, p<.05\right)$, Problemas de Comunicación con la madre $\left(\mathrm{F}_{2,565}=12.876, p<.001\right)$, Comunicación 
Tabla 1. Prueba de efectos inter-sujetos en función del nivel de victimización de pareja (sin victimización, victimización ocasional y victimización frecuente), del sexo (chico y chica) y de la interacción entre ambos grupos (victimización pareja*sexo)

\begin{tabular}{|c|c|c|c|c|}
\hline & Variable Dependiente & $\mathrm{F}$ & $p$ & $\eta^{2}$ \\
\hline \multirow{13}{*}{ Victimización pareja } & Autoconcepto Académico & 5.191 & .006 & .018 \\
\hline & Autoconcepto Social & 5.803 & .003 & .020 \\
\hline & Autoconcepto Emocional & 4.237 & .015 & .015 \\
\hline & Autoconcepto Familiar & 9.860 & .000 & .034 \\
\hline & Autoconcepto Físico & 3.291 & .038 & .012 \\
\hline & Comunicación Abierta Madre & 3.206 & .041 & .011 \\
\hline & Problemas Comunicación Madre & 12.876 & .000 & .044 \\
\hline & Comunicación Abierta Padre & 7.936 & .000 & .027 \\
\hline & Problemas Comunicación Padre & 5.671 & .004 & .020 \\
\hline & Ánimo depresivo & 13.210 & .000 & .045 \\
\hline & Soledad emocional & 8.911 & .000 & .031 \\
\hline & Evaluación de la red social & 4.675 & .010 & .016 \\
\hline & Satisfacción con la vida & 11.723 & .000 & .040 \\
\hline \multirow{13}{*}{ Sexo } & Autoconcepto Académico & .881 & .348 & .002 \\
\hline & Autoconcepto Social & 3.631 & .057 & .006 \\
\hline & Autoconcepto Emocional & 49.359 & .000 & .080 \\
\hline & Autoconcepto Familiar & .095 & .758 & .000 \\
\hline & Autoconcepto Físico & 55.155 & .000 & .089 \\
\hline & Comunicación Abierta Madre & 7.495 & .006 & .013 \\
\hline & Problemas Comunicación Madre & 1.204 & .273 & .002 \\
\hline & Comunicación Abierta Padre & 1.879 & .171 & .003 \\
\hline & Problemas Comunicación Padre & 2.338 & .127 & .004 \\
\hline & Ánimo depresivo & 33.893 & .000 & .057 \\
\hline & Soledad emocional & .011 & .917 & .000 \\
\hline & Evaluación de la red social & .139 & .709 & .000 \\
\hline & Satisfacción con la vida & 1.588 & .208 & .003 \\
\hline \multirow{13}{*}{ Victimización pareja* sexo } & Autoconcepto Académico & .446 & .641 & .002 \\
\hline & Autoconcepto Social & .739 & .478 & .003 \\
\hline & Autoconcepto Emocional & 2.169 & .115 & .008 \\
\hline & Autoconcepto Familiar & .601 & .548 & .002 \\
\hline & Autoconcepto Físico & .189 & .828 & .001 \\
\hline & Comunicación Abierta Madre & .593 & .553 & .002 \\
\hline & Problemas Comunicación Madre & .369 & .691 & .001 \\
\hline & Comunicación Abierta Padre & .094 & .911 & .000 \\
\hline & Problemas Comunicación Padre & .117 & .890 & .000 \\
\hline & Ánimo depresivo & 2.402 & .091 & .008 \\
\hline & Soledad emocional & .449 & .639 & .002 \\
\hline & Evaluación de la red social & .013 & .987 & .000 \\
\hline & Satisfacción con la vida & 1.492 & .226 & .005 \\
\hline
\end{tabular}

abierta con el padre $\left(\mathrm{F}_{2,565}=7.963, p<.001\right)$, Problemas de Comunicación con el padre $\left(\mathrm{F}_{2,565}=5.671, p<.01\right)$, Ánimo depresivo $\left(\mathrm{F}_{2,565}=13.210, p<.001\right)$, Soledad emocional $\left(\mathrm{F}_{2,565}=8.911, p<.001\right)$, Evaluación de la red social $\left(\mathrm{F}_{2,565}=4.675, p=.01\right)$ y Satisfacción con la vida $\left(\mathrm{F}_{2,565}=\right.$ $11.723, p<.001)$. Se observaron diferencias significativas entre chicos y chicas en Autoconcepto emocional $\left(\mathrm{F}_{1,565}=\right.$ $49.359, p<.001)$, Autoconcepto físico $\left(\mathrm{F}_{1,565}=55.155, p<\right.$ $.001)$, Comunicación abierta con la madre $\left(\mathrm{F}_{1,565}=7.495\right.$, $p<.01)$ y Ánimo depresivo $\left(\mathrm{F}_{1,565}=33.893, p<.001\right)$. No se encontraron diferencias significativas en la interacción.
Al constatarse efectos principales tanto del nivel de victimización como del sexo en algunas de las variables consideradas, se analizaron estos resultados. En la Tabla 2 se presentan las medias y desviaciones típicas en estas variables de los tres grupos de adolescentes establecidos en función de la frecuencia de la victimización. Al ser tres los grupos comparados, se realizaron pruebas a posteriori para analizar entre qué grupos estas diferencias eran significativas.

En la Tabla 2 se puede apreciar el peor ajuste psicosocial de los adolescentes victimizados de forma frecuente. Estos adolescentes difieren no sólo de los adolescentes no 
Tabla 2. Medias (y desviaciones típicas) en autoconcepto, comunicación padres-hijos, ánimo depresivo, soledad y satisfacción con la vida en función del nivel de victimización de pareja (sin victimización, victimización ocasional y victimización frecuente)

\begin{tabular}{llll}
\hline & Sin victimización & Victimización ocasional & Victimización frecuente \\
\hline Autoconcepto Académico & $63.13(22.25) \mathrm{a}$ & $59.46(21.74)$ & $52.85(20.95) \mathrm{b}$ \\
Autoconcepto Social & $73.08(15.68) \mathrm{b}$ & $77.03(14.33) \mathrm{a}$ & $73.29(16.14)$ \\
Autoconcepto Emocional & $55.65(19.97) \mathrm{a}$ & $53.29(18.80)$ & $48.92(20.30) \mathrm{b}$ \\
Autoconcepto Familiar & $85.36(15.01) \mathrm{a}$ & $82.89(15.91) \mathrm{a}$ & $75.04(19.31) \mathrm{b}$ \\
Autoconcepto Físico & $62.73(20.26)$ & $64.09(19.57)$ & $61.20(21.16)$ \\
Comunicación Abierta Madre & $44.06(9.08) \mathrm{a}$ & $42.35(9.32)$ & $40.64(11.11) \mathrm{b}$ \\
Problemas Comunicación Madre & $20.21(5.94) \mathrm{c}$ & $22.26(5.64) \mathrm{b}$ & $24.21(5.89) \mathrm{a}$ \\
Comunicación Abierta Padre & $40.19(10.00) \mathrm{a}$ & $38.23(9.91) \mathrm{a}$ & $34.29(11.26) \mathrm{b}$ \\
Problemas Comunicación Padre & $20.78(6.09) \mathrm{b}$ & $22.63(5.72) \mathrm{a}$ & $22.89(5.53) \mathrm{a}$ \\
Ánimo depresivo & $13.04(4.73) \mathrm{c}$ & $14.47(4.46) \mathrm{b}$ & $23.00(4.36) \mathrm{a}$ \\
Soledad emocional & $20.53(5.40) \mathrm{b}$ & $21.00(5.27) \mathrm{b}$ & $26.96(5.03) \mathrm{b}$ \\
Evaluación de la red social & $28.51(4.81)$ & $28.93(4.47) \mathrm{a}$ & $15.70(3.67) \mathrm{b}$ \\
Satisfacción con la vida & $18.08(3.87) \mathrm{a}$ & $17.97(3.88) \mathrm{a}$ & \\
\hline
\end{tabular}

Nota. $\mathrm{a}>\mathrm{b}>\mathrm{c}, \mathrm{p}<.05$; Prueba post-hoc Bonferroni

Tabla 3. Medias (y desviaciones típicas) en autoconcepto emocional, autoconcepto físico, comunicación abierta con la madre y ánimo depresivo en función del sexo de los adolescentes

\begin{tabular}{lll}
\hline & Chicos & Chicas \\
\hline Autoconcepto Emocional & $59.27(18.13)$ & $47.93(18.90)$ \\
Autoconcepto Físico & $70.86(16.70)$ & $56.34(20.19)$ \\
Comunicación Abierta Madre & $41.58(10.08)$ & $43.60(8.86)$ \\
Ánimo depresivo & $13.21(3.96)$ & $15.25(4.76)$ \\
& & \\
\hline
\end{tabular}

victimizados, sino también de los victimizados de forma ocasional, presentando menor Autoestima familiar, menor Comunicación abierta con la madre, menor Satisfacción con la vida y mayor Soledad emocional. En estas variables los adolescentes no victimizados y los victimizados de forma ocasional no difieren. Otra variable con diferencias significativas entre los adolescentes con victimización frecuente y ocasional es su Evaluación de la red social, siendo dicha evaluación más negativa en adolescentes con victimización frecuente. Estas variables serían, por tanto, especialmente relevantes en la diferenciación entre adolescentes en situación de victimización frecuente y de victimización ocasional. Por otra parte, se observan también diferencias significativas entre los tres grupos en las variables de Problemas de comunicación con la madre y Ánimo depresivo, siendo los adolescentes victimizados frecuentemente los que muestran más problemas de comunicación con la madre y mayor ánimo depresivo. Finalmente, se observan diferencias significativas entre los adolescentes victimizados frecuentemente y los adolescentes no victimizados en Autoconcepto académico, Autoconcepto emocional y Autoconcepto físico, mostrando en estas tres dimensiones peor autoconcepto los adolescentes victimizados frecuentemente en comparación con los no victimizados. Los adolescentes con victimización ocasional no difieren de ambos grupos en estas dimensiones del autoconcepto.

Por último, respecto a las diferencias en función del sexo, en la Tabla 3 puede observarse que los chicos muestran mayor Autoconcepto emocional y Autoconcepto físico que las chicas; mientras que las chicas muestran mayor Comunicación Abierta con la Madre y mayor Ánimo depresivo.

\section{Discusión}

En esta investigación se planteó como objetivo analizar el ajuste psicosocial de los adolescentes víctimas de violencia de pareja, considerando posibles diferencias en función de la frecuencia de victimización y el sexo del adolescente. Los resultados obtenidos muestran claramente un peor ajuste psicológico y social en los adolescentes cuya victimización es frecuente, no solo en comparación con 
aquellos no victimizados, sino también respecto a aquellos cuya victimización es ocasional. Estos resultados confirman la relación existente entre la victimización de pareja y el pobre ajuste psicosocial en los adolescentes (Exner-Cortens et al., 2013; Jouriles et al., 2009; Leen et al., 2013; Romito et al., 2013; Shorey et al., 2015), al tiempo que destacan la necesidad de diferenciar entre victimización frecuente y ocasional. Por otra parte, al no haber resultado significativa la interacción entre victimización y sexo del adolescente, este peor ajuste psicosocial se observa tanto en chicos como en chicas en situación de victimización frecuente.

Se constata, además, la elevada prevalencia de la violencia ocasional en parejas adolescentes al situarse la mayoría de los adolescentes, chicos y chicas, en el grupo de victimización ocasional. Estos resultados se sitúan en la línea de los obtenidos en investigaciones previas (Fernández-Fuertes y Fuertes, 2010; Viejo, 2014), pero resaltan además la existencia de un grupo menos numeroso, aunque más vulnerable, de chicos y chicas adolescentes víctimas frecuentes de la violencia de pareja. El peor ajuste psicosocial de los adolescentes victimizados de forma frecuente se ha constatado en esta investigación mediante diversos indicadores psicológicos y sociales. Así, en relación con la satisfacción con la vida y el ánimo depresivo, no solo se confirman las relaciones de estas variables con la victimización de pareja (Romito et al., 2013; Shorey et al., 2015), sino que además se constata un mayor ánimo depresivo y una menor satisfacción con la vida en las situaciones en las que los adolescentes sufren con mayor frecuencia la victimización de la pareja. Esta mayor frecuencia, probablemente unida a la coexistencia de varios tipos de violencia y a una mayor gravedad de las agresiones (Foshee y Reyes, 2011; Leen et al., 2013; Sears et al., 2007), afecta a estos adolescentes en mayor medida y podría ser un indicador de mayores dificultades de adaptación en otros ámbitos.

Los adolescentes victimizados por su pareja de forma frecuente muestran también mayores sentimientos de soledad y evalúan más negativamente su red social que los adolescentes no victimizados o victimizados de forma ocasional. Estos adolescentes es probable que tengan menor apoyo, confianza y cercanía con sus parejas, lo que incidiría en su mayor sentimiento de soledad puesto que la pareja es una importante fuente de apoyo durante la adolescencia (Musitu y Cava, 2003). Otra posible explicación es que esta mayor soledad sea debida a un menor apoyo del entorno social y un mayor aislamiento de la víctima como consecuencia de un mayor control ejercido por la pareja, una forma de violencia que no siempre los adolescentes identifican como tal (Díaz-Aguado y Carvajal, 2011).
Respecto al autoconcepto, se observan diferencias significativas en la mayoría de sus dimensiones, mostrando en todos los casos un peor autoconcepto los adolescentes cuya victimización es frecuente. Su peor autoconcepto académico puede ser indicativo de dificultades previas en el contexto escolar que podrían incrementar su vulnerabilidad a la violencia de pareja, o bien ser consecuencia de una relación de pareja absorbente y controladora que incidiría negativamente en su rendimiento académico. A este respecto, convendría desarrollar estudios longitudinales que permitieran analizar cómo estas variables se influyen mutuamente. Igualmente, su peor autoconcepto emocional podría ser tanto un factor de riesgo como una consecuencia de la victimización, un aspecto que también requerirá de futuros estudios. En el caso del autoconcepto familiar, los resultados obtenidos constatarían la relación existente entre la victimización de pareja en adolescentes y el contexto familiar donde se desarrolla el adolescente (Foshee et al., 2015; Kast et al., 2015; Vézina y Herbert, 2007). Unas relaciones familiares caracterizadas por el afecto, la confianza y el apoyo, además de constituir un modelo positivo de relaciones interpersonales para los hijos, pueden también favorecer en ellos un autoconcepto familiar positivo $\mathrm{y}$, a través de este autoconcepto positivo, propiciar que sean capaces de desarrollar relaciones de pareja más positivas.

La importancia de algunas características familiares, tales como la violencia intrafamiliar o la carencia de afecto y normas, como factores de riesgo en relación con la violencia y victimización en parejas adolescentes ha sido señalada en trabajos previos (Foshee et al., 2015; González-Ortega, Echeburúa y Corral, 2008; Kast et al., 2015). Los resultados de esta investigación sugieren también la importancia específica que la falta de comunicación adecuada entre padres e hijos puede tener como un posible factor de riesgo para la victimización de pareja en los hijos. Los adolescentes que sufren esta victimización con mayor frecuencia manifiestan tener una comunicación menos abierta y positiva con ambos progenitores, así como también más problemas de comunicación con la madre. En todas las dimensiones de la comunicación padres-hijos analizadas, los adolescentes no victimizados son los que mantienen mejor comunicación con ambos padres. Esta mejor comunicación familiar podría ayudar a estos adolescentes a afrontar mejor y con mayor confianza sus primeras relaciones de pareja.

Por último, y aunque la frecuencia de la victimización no interactúa con el sexo del adolescente en relación con su ajuste psicosocial, sí se han observado diferencias entre chicos y chicas en algunas variables. Los resultados obtenidos confirman el mayor autoconcepto físico y emocional 
de los chicos (Revuelta, Esnaola y Goñi, 2013) y el mayor ánimo depresivo de las chicas (Povedano, Jiménez, Moreno, Amador y Musitu, 2012). Algunas diferencias en los patrones de socialización y en determinadas características culturales podrían explicar, en cierta medida, estas diferencias. Por otra parte, los diferentes estilos de socialización utilizados por los padres según el sexo de los hijos (Garaigordobil y Aliri, 2012), podrían contribuir también a explicar que la comunicación sea más abierta entre madres e hijas, un resultado que ha sido obtenido también en otros estudios (Cava et al., 2014).

Ciertamente, este estudio tiene también algunas limitaciones importantes. En primer lugar, se trata de un estudio transversal que no permite establecer relaciones de causalidad, por lo que únicamente pueden afirmarse relaciones entre variables. En futuras investigaciones sería necesario realizar estudios longitudinales que permitan comprender mejor cómo la victimización afecta al ajuste psicológico y social de los adolescentes y también como determinadas dificultades de ajuste psicosocial en los adolescentes pueden incrementar su vulnerabilidad a sufrir situaciones de violencia de pareja. Estos estudios longitudinales permitirán también analizar con mayor profundidad la relevancia de la frecuencia de las conductas violentas en las parejas adolescentes, así como su posible evolución en el tiempo y su potencial utilidad para identificar adolescentes en situación de mayor riesgo. Por otra parte, además de la frecuencia de la victimización, un análisis más completo de esta problemática requeriría considerar también las motivaciones que chicos y chicas atribuyen a las agresiones, su percepción de las mismas y sus creencias sobre las relaciones de pareja.

A pesar de estas limitaciones, esta investigación aporta resultados interesantes sobre la violencia en parejas adolescentes y su relación con el ajuste psicosocial de los adolescentes. En este sentido, se aportan datos que sugieren la necesidad de distinguir entre adolescentes en situación de victimización ocasional y aquellos cuya victimización es frecuente. Estos últimos presentan claramente un peor ajuste psicosocial y podrían ser más vulnerables a ser víctimas de violencia de pareja en la edad adulta, así como también es más probable que presenten durante la adolescencia dificultades de adaptación en otros ámbitos. Las intervenciones centradas en reducir la violencia de pareja deberían centrarse prioritariamente en estos adolescentes, ayudándoles a reflexionar sobre las consecuencias de la violencia y aportándoles recursos personales y sociales que les ayuden a establecer relaciones de pareja más saludable.

\section{Referencias}

Atienza F. L., Pons, D., Balaguer, I., y García-Merita, M. (2000). Propiedades psicométricas de la escala de satisfacción con la vida en adolescentes. Psicothema, 12, 314-320.

Barnes, H. L., y Olson, D.H. (1985). Parent-adolescent communication and the circumplex model. Child Development, 56, 438-447.

Borges, A., Prieto P., Ricchetti G., Hernández-Jorge C., y RodriguezNaveiras, E. (2008). Validación cruzada de la factorización del Test UCLA de Soledad. Psicothema, 20, 924-927.

Buelga, S., Iranzo, B., Cava, M. J., y Torralba, E. (2015). Psychological profile of adolescent cyberbullying aggressors. Revista de Psicología Social-International Journal of Social Psychology, 30, 1-25.

Cava, M. J., Buelga, S., y Musitu, G. (2014). Parental communication and life satisfaction in adolescence. Spanish Journal of Psychology, 17, E98.

Cava, M. J., Murgui, S., y Musitu, G. (2008). Diferencias en factores de protección del consumo de sustancias en la adolescencia temprana y media. Psicothema, 29, 389-395.

Coleman, J. C., y Hendry, L. B. (2003). Psicología de la adolescencia. Madrid: Morata.

Cornelius, T. L., y Resseguie, N. (2007). Primary and secondary prevention programs for dating violence: A review of the literature. Aggression and Violent Behavior, 12, 364-375.

Cortés-Ayala, L., Flores, M., Bringas, C., Rodríguez-Franco, L., LópezCepero, J., y Rodríguez, J. (2015). Relación de maltrato en el noviazgo de jóvenes mexicanos. Análisis diferencias por sexo y nivel de estudios. Terapia Psicológica, 33, 5-12.

Cuevas, C. A., Sabina, C., y Bell, K.A. (2014). Dating violence and interpersonal victimization among national simple of Latino youth. Journal of Adolescent Health, 55, 564-570.

Díaz-Aguado, M. J., y Carvajal, I. (Dirs.) (2011). Igualdad y prevención de la violencia de género en la adolescencia y la juventud. Madrid: Ministerio de Sanidad, Igualdad y Servicios Sociales.

Diener, E., Emmons, R., Larsen, R. J., y Griffin, S. (1985). The Satisfaction with Life Scale. Journal of Personalitiy Assessment, 49, 71-75.

Estévez, E., Martínez, B., y Musitu, G. (2006). La autoestima en adolescentes agresores y víctimas en la escuela: La perspectiva multidimensional. Psychosocial Intervention, 15, 223-232.

Exner-Cortens, D., Eckenrode, J., y Rothman, E. (2013). Longitudinal associations between teen dating violence victimization and adverse health outcomes. Pediatrics, 131, 71-78.

Expósito, F., y Moya, M. C. (1999). Soledad y Apoyo Social. Revista de Psicología Social, 2-3, 319-339.

Fernández-Fuertes, A. A., y Fuertes, A. (2010). Physical and psychological aggression in dating relationships of Spanish adolescents. Motives and consequences. Child Abuse \& Neglect, 34, 183-191.

Fernández-Fuertes, A. A., Fuertes, A., y Pulido, R. F. (2006). Evaluación de la violencia en las relaciones de pareja de los adolescentes. Validación del Conflict in Adolescent Dating Relationships Inventory (CADRI)-versión española. International Journal of Clinical and Health Psychology, 6, 339-358.

Foshee, V. A., Reyes, L. M., Tharp, A. T., Chang, L.Y., Ennett, S.T., Simon, T.R., y Suchindran, C. (2015). Shared longitudinal predictors of physical peer and dating violence. Journal of Adolescent Health, 56, 106-112.

Foshee, V., y Reyes, H. (2011). Dating abuse: Prevalence, consequences and causes. En J.R. Levesque (Eds.), Encyclopedia of adolescence (pp. 602-615). New York: Springer.

Furman, W., Low, S., y Ho, M. J. (2009). Romantic experience and psychosocial adjustment in middle adolescence. Journal of Clinical Child \& Adolescent Psychology, 38, 75-90.

Garaigordobil, M., y Aliri, J. (2012). Parental socialization styles, parents' educational level and sexist attitudes in adolescence. The Spanish Journal of Psychology, 15, 592-603.

García, F., y Musitu, G. (1999). AF-5: Autoconcepto Forma-5. Madrid: TEA.

García, F., Musitu, G., Riquelme, E., y Riquelme, P. (2011). A confirmatory factor analysis of the "Autoconcepto Forma 5" questionnaire in 
young adults from Spain and Chile. Spanish Journal of Psychology, $14,648-658$.

González-Ortega, I., Echeburúa, E., y Corral, P. (2008). Variables significativas en las relaciones violentas en parejas jóvenes: una revisión. Psicología Conductual, 16, 207-225.

González-Pienda, J. A., Nuñez, J. C., Álvarez, L. Roces, C., GonzálezPumariega, S., González, P.,... Bernardo, A. (2003). Adaptabilidad y cohesión familiar, implicación parental en conductas autorregulatorias, autoconcepto del estudiante y rendimiento académico. Psicothema, $15,471-477$.

Herrero, J., y Meneses, J. (2006). Short Web-based versions of the perceived stress (PSS) and Center for Epidemiological Studies-Depression (CESD) Scales: a comparison to pencil and paper responses among Internet users. Computers in Human Behavior, 22, 830-848.

Jiménez, T. I., Estévez, E., Musitu, G., y Murgui, S. (2007). Comunicación familiar y comportamientos delictivos en la adolescencia: el doble rol mediador de la autoestima. Revista Latinoamericana de Psicología, 39, 473-485.

Jouriles, E. N., Garrido, E., Rosenfield, D., y McDonald, R. (2009). Experiences of psychological and physical aggression in adolescent romantic relationships: Links to psychological distress. Child Abuse \& Neglect, 33, 451-460.

Kast, N. R., Eisenberg, M. E., y Sieving, R. E. (2015). The role of parent communication and connectedness in dating violence victimization among Latino adolescents. Journal of Interpersonal Violence. Published online before print, February, 24.

Leen, E., Sorbring, E., Mawer, M., Holdsworth, E., Helsing, B., y Bowen, E. (2013). Prevalence, dynamic risk factors and the efficacy of primary interventions for adolescent dating violence: An international review. Aggression and Violent Behavior, 18, 159-174.

Micó-Cebrián, P., y Cava, M. J. (2014). Sensibilidad intercultural, empatía, autoconcepto y satisfacción con la vida en alumnos de educación primaria. Infancia y Aprendizaje, 37, 342-367.

Muñoz-Rivas, M.J., Graña, J. L., O’Leary, K. D., y González, M. P. (2007). Aggression in adolescent dating relationships: Prevalence, justification, and health consequences. Journal of Adolescent Health, 40, 298-304.

Musitu, G., y Cava, M. J. (2003). El rol del apoyo social en el ajuste de los adolescentes. Psychosocial Intervention, 12, 179-192.

O’Leary, K. D., Slep, A. M., Avery-Leaf, S., y Cascardi (2008). Gender differences in dating aggressions and victimization among multiethnic high school students. Journal of Adolescent Health, 42, 473-479.

Ortega, R., y Sánchez, V. (2011). Juvenile dating and violence. En C.P. Monks y I. Coyne (Eds.), Bullying in different contexts (pp.113-136). London: Cambridge University Press.

Povedano, A., Cava, M. J., Monreal, M. C., Varela, R., y Musitu, G. (2015). Victimization, loneliness, overt and relational violence at the school from a gender perspective. International Journal of Clinical and Health Psychology, 15, 44-51.

Povedano, A., Jiménez, T. I., Moreno, D., Amador, L. V., y Musitu, G. (2012). Relación del conflicto y la expresividad familiar con la victimización en la escuela: el rol de la autoestima, la sintomatología depresiva y el género de los adolescentes. Infancia y Aprendizaje, 35, 421-432.

Radloff, L. S. (1977). The CES-D scale: A self-report depression scale for research in the general population. Applied Psychological Measurement, 1, 385-401.

Revuelta, L., Esnaola, I., y Goñi, A. (2013). El autoconcepto físico determinante de la actividad física en la adolescencia. Psicología Conductual, 21, 581-601.

Rey-Anacona, C. A. (2013). Prevalencia y tipos de maltrato en el noviazgo en adolescentes y adultos jóvenes. Terapia Psicológica, 31, 143-154.

Rey-Anacona, C. A., Mateus-Cubides, A. M., y Bayona-Arévalo, P.A. (2010). Malos tratos ejercidos por adolescentes durante el noviazgo: diferencias por sexo. Revista Mexicana de Psicología, 27, 169-181.

Romito, P., Beltramini, L., y Escribà-Agüir, V. (2013). Intimate partner violence and mental health among Italian adolescents: gender similarities and differences. Violence Against Women, 19, 89-106.
Russell, D., Peplau, L. A., y Cutrona, C.E. (1980). The revised UCLA loneliness Scale: Concurrent and discriminant validity evidence. Journal of Personality and Social Psychology, 39, 472-480.

Saldivia, C., y Vizcarra, B. (2012). Consumo de drogas y violencia en el noviazgo en estudiantes universitarios del sur de Chile. Terapia Psicológica, 30, 43-49.

Sears, H. A., Byers, E. S., y Price, E. L. (2007). The co-ocurrence of adolescent boys' and girls' use of psychologically, physically, and sexually abusive behaviors in their dating relationships. Journal of Adolescence, 30, 487-504.

Shorey, R. C., Rhatigan, D. L., Fite, P. J., y Stuart, G. L. (2011). Dating violence victimization and alcohol problems: An examination of the stress-buffering hypothesis for perceived support. Partner Abuse, 2, 31-45.

Shorey, R.C., Seavey, A. E., Brasfield, H., Febres, J., Fite, P. J., y Stuart, G. L. (2015). The moderating effect of social support from a dating partner on the association between dating violence victimization and adjustment. Violence Against Women, 21, 460-477.

Vézina, J., y Hébert, M. (2007). Risk factors for victimization in romantic relationships of young women: A review of empirical studies and implications for prevention. Trauma, Violence, \& Abuse, 8, 33-66.

Viejo, C. (2014). Violencia física en las relaciones sentimentales adolescentes: hacia la comprensión del fenómeno. Infancia y Aprendizaje, 37, 785-815.

Viejo, C., Monks, C. P., Sánchez, V., y Ortega-Ruiz, R. (2015). Physical dating violence in Spain and the United Kingdom and the importance of relationship quality. Journal of Interpersonal Violence, 1-23.

Wolfe, D. A., Scott, K., Reitzel-Jaffe, D., Wekerle, C., Grasley, C. y Pittman, A. L. (2001). Development and validation of the conflict in adolescent dating relationships inventory. Psychological Assessment, 13, 277-293. 